\title{
ARTIGOS
}

Submetido 28.11.2019. Aprovado 07.10.2020

Avaliado pelo sistema double blind review. Editor Científico convidado: Francisval Carvalho

Versão original

DOI: http://dx.doi.org/10.1590/So034-759020210202

\section{GOVERNANÇA CORPORATIVA E CONEXÕES POLÍTICAS NAS PRÁTICAS ANTICORRUPÇÃO}

\author{
Corporate governance and political connections in anti-corruption practices \\ Gobierno corporativo y conexiones políticas en prácticas anticorrupción
}

\section{SIRLENE KOPROWSKI ${ }^{1}$}

sirlene.koprowski@unochapeco.edu.br

oooo-0002-6661-2697

\section{VIVIANE KREIN ${ }^{1}$}

vivianekrein@unochapeco.edu.br

0000-0002-2768-6992

\section{SADY MAZZIONI ${ }^{1}$}

sady@unochapeco.edu.br

oooo-0002-8976-6699

\section{CRISTIAN BAÚ DAL MAGRO ${ }^{1}$}

crisbau@unochapeco.edu.br

0000-0002-7609-5806

1 Universidade Comunitária da Região de Chapecó, Programa de Pós-Graduação em Ciências Contábeis e Administração, Chapecó, SC, Brasil

\section{RESUMO}

O objetivo do estudo é analisar se mecanismos específicos de governança corporativa e as conexões políticas influenciam a evidenciação voluntária de práticas anticorrupção em empresas listadas na [B]3. $O$ índice de evidenciação foi obtido a partir da relação do número de citações das palavras-chave com o número de páginas dos relatórios de sustentabilidade. A análise foi realizada por meio de regressão linear múltipla e considerou 740 observações dos exercícios de 2016 e 2017. Os resultados indicaram que o tamanho e o percentual de membros externos no Conselho de Administração (CA), auditoria big four, tamanho do comitê de auditoria e a participação acionária do governo influenciaram positivamente os níveis de evidenciação de práticas anticorrupção. Por sua vez, a presença de políticos no CA conduz para aumento da assimetria informacional ao revelar menor nível de divulgação, que, na sua extensão, representa menor compromisso com as práticas anticorrupção.

PALAVRAS-CHAVE | Governança corporativa, vínculos políticos, divulgação, combate à corrupção, auditoria.

\section{ABSTRACT}

The objective of this study is to analyze whether specific corporate governance mechanisms and political connections influence the voluntary disclosure of anti-corruption practices in the companies listed in the Brazilian stock exchange $[B] 3$. The disclosure index was obtained from the relationship between the number of citations of the keywords and the number of pages in the sustainability reports. The analysis was performed using multiple linear regression, considering 740 observations from financial years 2016 and 2017. The results indicate that the size and percentage of external members on the board of directors, Big Four audits, size of the audit committee, and participation of government shareholding positively influence the levels of disclosure of anti-corruption practices. In turn, the presence of politicians on the board of directors leads to an increase in information asymmetry due to a lower level of disclosure, which, by extension, represents less commitment to anti-corruption practices.

KEYWORDS / Corporate governance, political links, disclosure, anti-corruption, audit.

\section{RESUMEN}

El objetivo del estudio es analizar si los mecanismos específicos de gobierno corporativo y las conexiones políticas influyen en la divulgación voluntaria de las prácticas anticorrupción en las empresas que cotizan en la $[B] 3$. El índice de divulgación se obtuvo de la relación entre el número de citas de las palabras clave y el número de páginas de los informes de sostenibilidad. El análisis se realizó mediante regresión lineal múltiple y consideró 740 observaciones de los ejercicios 2016 y 2017. Los resultados indicaron que el tamaño y el porcentaje de miembros externos en el consejo de administración, la auditoría bif four, el tamaño del comité de auditoría y la participación accionaria del gobierno influyeron positivamente en los niveles de divulgación de las prácticas anticorrupción. A su vez, la presencia de políticos en el consejo de administración conduce a un aumento de la asimetría de la información al revelar un menor nivel de divulgación, que, en su extensión, representa un menor compromiso con las prácticas anticorrupción. PALABRAS CLAVE / Gobierno corporativo, vínculos políticos, divulgación, anticorrupción, auditoría. 


\section{INTRODUÇÃO}

No Brasil, o baixo cumprimento das leis e a fiscalização deficitária do governo encorajam as empresas às práticas antiéticas (Halter, Arruda, \& Halter, 2009). A Operação Lava Jato revelou um esquema envolvendo grandes empresas e a esfera política nacional, considerado pelo World Economic Forum como um dos maiores escândalos de corrupção no Brasil (Padula \& Albuquerque, 2018).

Para coibir tais comportamentos, o governo brasileiro promoveu esforços na aprovação da Lei n. 12.846/2013 (Lei Anticorrupção) e do Decreto n. 8.420/2015, que sinalizaram a necessidade de compliance como legislação indutiva, a partir do marco normativo da Governança Corporativa (GC), com orientações sobre os mecanismos internos de integridade, auditoria e incentivo à denúncia de irregularidades, aplicação dos códigos de ética e de conduta pelas empresas (Lei n. 12.846, 2013).

A GC ganhou destaque para promover a transparência e regulação das práticas anticorrupção nas companhias abertas, sendo também um tema central da Responsabilidade Social Corporativa (RSC) (Aguilera, Rupp, Williams, \& Ganapathi, 2007; Jamali, Safieddine, \& Rabbath, 2008; Kaymak \& Bektas, 2017).

Os mecanismos de GC são fundamentais na detecção e no monitoramento da corrupção corporativa (Agyei-Mensah, 2017; Frías-Aceituno, Rodríguez-Domínguez, \& García-Sánchez, 2014; Malagueño, Albrecht, Ainge, \& Stephens, 2010; Na, Kang, \& Kim, 2018; Owolabi, 2011; Wu, 2005), e o seu combate é um importante atributo da RSC (Branco \& Delgado, 2012; Hills, Fiske, \& Mahmud, 2009; Hoi \& Lin, 2012). Desse modo, o combate à corrupção precisa ser suportado pelos mecanismos de GC por meio das práticas de RSC (Malagueño et al., 2010).

A Teoria da Agência preconiza que a separação entre a propriedade (proprietários/acionistas) e o controle da empresa (gestores contratados) produz conflitos e custos de agência (Jensen \& Meckling, 1976). À medida que aumenta a dispersão da propriedade, maiores são os conflitos de agência entre controladores e demais partes (Depoers, 2000). Conflitos de agência envolvem a possibilidade de o acionista controlador expropriar riquezas de outros acionistas e os problemas de assimetria de informações (Jensen \& Meckling, 1976), que, na sua extensão, influenciam a evidenciação voluntária (Chow \& Wong-Boren, 1987).

Em ambientes de elevada dispersão do capital, a exemplo dos Estados Unidos da América, são conferidos poderes elevados aos executivos e menores aos acionistas, o que gera problemas entre acionistas e executivos (Nassif \& Souza, 2013). Contudo, as empresas brasileiras apresentam elevada concentração de propriedade, e o principal conflito de agência ocorre entre acionistas majoritários e minoritários (Dami, Rogers, \& Ribeiro, 2007).

Nas empresas em que o controle acionário é mais disperso (menos concentrado), há a necessidade de maior divulgação de informações (Raffournier, 1995) para resolver os problemas de assimetria informacional (Jensen \& Meckling, 1976). Por sua vez, acionistas com participação acionária relevante integram a gestão e possuem acesso privilegiado às informações (Leuz, 2006).

A GC apresenta mecanismos que permitem reduzir os conflitos de agência entre principal e agente ou entre controlador e minoritários (Silveira \& Barros, 2008), agindo na redução dos custos de agência (Dami et al., 2007).

Complementarmente, as firmas usam a RSC como extensão dos mecanismos de GC no intuito de divulgar informações voluntárias que minimizem a assimetria informacional. Contudo, a GC é impactada pelos fatores sociais e políticos do ambiente, tendo o Conselho de Administração (CA) condições de modificar a atuação na RSC (Jensen \& Meckling, 1976).

Por sua vez, as conexões políticas são intrínsecas à estrutura de propriedade e aos mecanismos de GC, pela participação de indivíduos com expertise política no CA das empresas, trazendo benefícios e vantagens competitivas, como o acesso às fontes informacionais e financeiras privilegiadas que contribuem para a redução da assimetria informacional e dos conflitos de agência (Camilo, Marcon, \& Bandeira-de-Mello, 2012).

As conexões políticas apresentam-se como aliadas da GC no interesse de fortalecer a legitimidade da imagem corporativa perante as partes interessadas, por meio da RSC. A divulgação voluntária de práticas anticorrupção parece representar uma forma coerente de a empresa com conexões políticas criar confiança com as partes interessadas (Said, Zainuddin, \& Haron, 2009).

A GC e as conexões políticas exercem pressão coerciva para que as empresas evidenciem informações sobre práticas anticorrupção. É importante estudar conjuntamente o impacto da GC e das conexões políticas na RSC, tendo em vista que se assemelham na forma com que buscam atender as partes interessadas e os conflitos de agência. Estudos anteriores têm utilizado o arcabouço da Teoria da Agência para revelar que, no campo da GC, a conexão política utilizada como equilíbrio de poder agrava os conflitos de interesses entre majoritários e minoritários (Ding, Jia, Wilson, \& Wu, 2015; Huang \& Zhao, 2016; Pan \& Tian, 2017).

Diante do exposto, emerge o seguinte problema: qual a influência de mecanismos da GC e das conexões políticas na evidenciação voluntária de práticas anticorrupção? O objetivo 
do estudo é analisar se mecanismos específicos de GC e as conexões políticas influenciam a evidenciação voluntária de práticas anticorrupção em empresas listadas na $[B]^{3}$.

Justifica-se o ambiente de estudo, pois empresas que atuam em mercados emergentes apresentam um ambiente propício para a corrupção (Krishnamurti, Shams, \& Velayutham, 2018). No período de análise, o Brasil ocupava a $79^{\underline{a}}$ e $96^{a}$ posições no ranking da transparência mundial, respectivamente em 2016 e 2017, no total de 180 países pesquisados (Transparência Internacional, 2017), caracterizando um ambiente de negócios favorável ao estudo da corrupção. Dada a vulnerabilidade do País no contexto global da transparência, a GC e as conexões políticas têm o potencial de influenciar o engajamento das empresas nas práticas de combate à corrupção e sua respectiva evidenciação.

Do ponto de vista da reputação corporativa, a evidenciação de práticas anticorrupção amplia a RSC e promove a legitimidade perante as partes interessadas (Etxeberria \& Odriozola, 2018). Do ponto de vista gerencial, minimiza os conflitos de agência (Jensen \& Meckling, 1976), e as estratégias de prevenção da corrupção integram a RSC (Hills et al., 2009), compondo a agenda de políticos, gestores, acionistas e estudiosos (Hoi \& Lin, 2012).

\section{REFERENCIAL TEÓRICO}

\section{RSC e práticas anticorrupção}

As iniciativas de RSC exigem das empresas comunicação e transparência com as partes interessadas sobre os esforços na luta contra a corrupção (Branco \& Delgado, 2012). Essa luta oferece oportunidade estratégica para os programas de RSC, ao abordar questão intrinsecamente ligada aos interesses corporativos e sociais (Hills et al., 2009).

A rejeição da corrupção é inerente à empresa com RSC, ao ser considerada incompatível com o desenvolvimento sustentável, pelos danos sociais, econômicos e ambientais que provoca (Branco \& Delgado, 2012). Contudo, a corrupção continua sendo questão social negligenciada entre as prioridades da RSC (Hills et al., 2009).

A corrupção no setor privado é caracterizada pelo abuso da autoridade na obtenção de benefício privado (Rodriguez, Siegel, Hillman, \& Eden, 2006), ocorrendo pela oferta de brindes, uso ilegítimo ou negociação de informação para benefício pessoal ou da organização (Halter et al., 2009).

As organizações são encorajadas a implementar estratégias para combater a corrupção, que pode estar relacionada ao uso indevido da contabilidade, espionagem empresarial, manipulação de informações, compras para benefício pessoal, pagamentos de propinas e roubo de ativos (Hills et al., 2009).

Ao investigar empresas da Malásia e da Indonésia, Joseph et al. (2016) observaram incipiente divulgação de práticas anticorrupção na RSC, sendo os códigos de conduta e as práticas de denúncia os itens mais relatados. No Brasil, algumas empresas privadas tornaram-se conscientes dos riscos envolvidos em procedimentos não éticos e iniciaram a adoção de práticas especificas de GC para o tema (Halter et al., 2009), sendo a Lei n. 12.846/2013 um fator de estímulo.

\section{GC e RSC em práticas anticorrupção}

Sob a égide da GC, as empresas são incentivadas a promover a ética, imparcialidade, prestação de contas, transparência e responsabilidade em todas as suas relações. Portanto, as empresas devem manter suas atividades em sintonia com as aspirações éticas e legais da sociedade (Jamali et al., 2008).

A abordagem da RSC equilibra as necessidades de diferentes grupos com os objetivos dos acionistas, incorporando preocupações sociais, ambientais e públicas na GC. Percebe-se convergência nos temas de GC e RSC, pois ambos buscam garantir a resistência dos negócios (Aguilera et al., 2007).

A GC representa um pilar para a sustentabilidade da RSC, e os países em desenvolvimento devem aumentar a vigilância e a capacidade dos sistemas regulatórios e judiciais sobre a GC e as pressões institucionais coercitivas na adoção da RSC Uamali et al., 2008). A RSC é tida como extensão da firma para promover a eficácia da GC, garantindo a sustentabilidade pelas práticas empresariais que promovam a responsabilidade e transparência aos acionistas e a sociedade (Jo \& Harjoto, 2012).

A GC afeta positivamente o engajamento da empresa na RSC (Jo \& Harjoto, 2012) e exerce um papel de catalisador para romper o ciclo vicioso de corrupção nas organizações $(\mathrm{Wu}$, 2005), constituindo-se instrumento de supervisão para garantir comportamento ético e coerente da gestão (Frías-Aceituno et al., 2014).

A corrupção atua como um dreno nos recursos das empresas e cria desincentivo à GC (Bishara, 2011), cujo combate dever ser promovido por controles que inibam práticas corruptas (Castro, Amaral, \& Guerreiro, 2019). Principalmente em mercados emergentes, os sistemas de GC menos desenvolvidos são mais suscetíveis à exploração por indivíduos corruptos (Bishara, 2011).

$\mathrm{H}_{1}$ : Os mecanismos de GC aumentam a RSC das empresas na evidenciação de práticas anticorrupção. 
A GC desempenha papel essencial na manutenção da integridade corporativa e na gestão do risco de fraude e corrupção nas companhias, constituindo-se a comunicação aos seus stakeholders em subsídio para a prestação de contas e implantação de medidas robustas para limitar e impedir a corrupção (Agyei-Mensah, 2017).

É responsabilidade do CA aprovar políticas anticorrupção com orientações sobre atividades de RSC e estabelecer mecanismos para prevenir o comportamento antiético (Bierstaker, 2009). Além disso, o maior número de membros no CA facilita o monitoramento e controle das práticas ilícitas (Agyei-Mensah, 2017).

A literatura preconiza que as características do CA influenciam a eficácia da GC. Nessa abordagem, o CA independente representa os interesses dos acionistas, prevenindo o envolvimento dos gestores em atos de suborno ( $\mathrm{Wu}, 2005)$. Os gestores de empresas com supervisão do CA com membros independentes aumentam a seriedade na aplicação e adoção das políticas de combate à corrupção (Healy \& Serafeim, 2015).

Evidências empíricas indicaram que a independência (Kaymak \& Bektas, 2017; Khan, Muttakin, \& Siddiqui, 2013) e o tamanho do CA (Kaymak \& Bektas, 2017) afetam positivamente as práticas de RSC. Os achados prévios também revelaram que a independência do CA (Agyei-Mensah, 2017; Donnelly \& Mulcahy, 2008) e o tamanho do CA (Donnelly \& Mulcahy, 2008) impactam positivamente a evidenciação voluntária pelas organizações, desempenhando papel importante na manutenção da integridade empresarial e no combate à corrupção. Frías-Aceituno et al. (2014) identificaram que a GC reforça o controle de atos de suborno e corrupção pela combinação dos diferentes mecanismos no ambiente corporativo.

$\mathrm{H}_{1 \mathrm{a}}$ : A presença de membros externos e o tamanho do Conselho de Administração aumentam a RSC das empresas na evidenciação de práticas anticorrupção.

A existência de comitê de auditoria com uma maior proporção de conselheiros independentes reduz os custos de agência e melhora o controle interno, conduzindo a maior qualidade das divulgações. Ao estudar empresas da Malásia, Said et al. (2009) indicaram que o comitê de auditoria se relaciona positivamente com a divulgação de RSC. Complementarmente, Khan et al. (2013) confirmaram o efeito positivo do comitê de auditoria nas práticas de RSC. 0 comitê de auditoria desempenha papel eficaz na atuação da GC, a exemplo das evidências de Ho e Wong (2001) de que a existência do comitê de auditoria está significativa e positivamente relacionada com a extensão da divulgação voluntária.
$\mathrm{H}_{1 \mathrm{~b}}$ : A presença de membros externos e o tamanho do comitê de auditoria aumentam a RSC das empresas na evidenciação de práticas anticorrupção.

Além dos esforços internacionais e exigências legais em nível de país, os padrões de contabilidade e auditoria alicerçados aos princípios da GC culminam na qualidade e integridade das organizações que mitigam e coíbem práticas corruptas. A contabilidade é um mecanismo essencial de transparência, cujo papel consiste em demonstrar as operações, refletidas pela realidade de maneira precisa e comparável, e, em contrapartida, a auditoria tem a prerrogativa de garantir a precisão dessas informações aos seus usuários (Owolabi, 2011).

Outro aspecto relevante da GC é a auditoria externa, constituindo-se em dispositivo de controle no monitoramento e detecção do suborno (Na et al., 2018), com papel de assegurar a confiabilidade das informações, diminuindo o risco informacional (Malagueño et al., 2010).

No âmbito dos países, Malagueño et al. (2010) investigaram 57 jurisdições e indicaram que aperfeiçoar a qualidade da contabilidade e da auditoria contribui para diminuir a corrupção. $\mathrm{Na}$ esfera organizacional, $\mathrm{Na}$ et al. (2018) evidenciaram que elementos da GC impactaram a prevenção da corrupção em empresas do Brasil, Rússia, Índia e China (BRIC).

A qualidade da contabilidade e auditoria também foi considerada estímulo para mitigar a corrupção, no estudo de Owolabi (2011) em 13 países africanos. As quatro grandes firmas mundiais de auditoria (big four) têm sido exigentes sobre as normas anticorrupção, fazendo emergir fortemente o combate à corrupção nas empresas (Healy \& Serafeim, 2015).

H1c: As firmas de auditoria big four aumentam a RSC das empresas na evidenciação de práticas anticorrupção.

\section{Conexões políticas e a RSC em práticas anticorrupção}

As conexões políticas são fenômenos mundiais em que os grupos empresariais se conectam com membros do governo de diversas formas: o governo sendo acionista de empresas privadas; empresário sendo político ou indicado para ocupar cargo de governo; políticos ou ex-políticos ocupando CA ou direção executiva de empresas privadas; empresas com doações para campanha de políticos (Claessens, Feijen, \& Laeyen, 2008).

As conexões políticas são associadas ao lobbying, em que prevalece a transmissão de informações privilegiadas para as empresas privadas (Correia, 2014). As empresas estreitam os vínculos políticos com o objetivo de reduzir incertezas, facilitar acesso ao crédito, ter menor controle regulatório, obter benefícios 
fiscais (Camilo et al., 2012; Huang \& Zhao, 2016; Wang, Chen, Chin, \& Zheng, 2017) e outros recursos controlados pelo Estado. As conexões políticas desempenham papel importante em economias com fraca regulamentação, alto nível de corrupção e instituições com fraco poder (Polsiri \& Jiraporn, 2012). As participações governamentais conduzem à RSC, pela responsabilidade do governo com a transparência das empresas de capital aberto (Nasir \& Abdullah, 2004). Desse modo, criar maior confiança entre as partes interessadas torna-se fator primordial para as empresas politicamente conectadas (Martynova \& Renneboog, 2009).

Evidências empíricas confirmaram que empresas com controle acionário do governo estão positivamente relacionadas com as divulgações de RSC (Khan et al., 2013; Said et al., 2009) e empresas com conexões políticas pela participação acionária estão interessadas na manutenção da reputação corporativa (Huang \& Zhao, 2016).

Achados apontam que a reputação social da empresa está positivamente relacionada à divulgação de informações na área de anticorrupção (Etxeberria \& Odriozola, 2018), e a RSC tem sido uma necessidade política constante, conduzindo empresas conectadas politicamente a aumentar investimentos nesse campo (Huang \& Zhao, 2016).

H2a: A participação acionária do governo aumenta a RSC das empresas na evidenciação de práticas anticorrupção.

A conexão política também é estabelecida pela presença de políticos no CA de empresas privadas, apresentando interesses compensatórios para a empresa e o indivíduo, sendo: a) empresas estreitam vínculos com interesse de obter recursos facilitados, minimizar o controle regulatório e obter benefícios fiscais; e b) políticos possuem interesses privados (Camilo et al., 2012; Chaney, Faccio, \& Parsley, 2011; Guedhami, Pittman, \& Saffar, 2014).

A presença de políticos no CA prevalece em ambientes com alta corrupção (Boubakri, Cosset, \& Saffar, 2008) e, no decorrer das relações entre empresas e governo, o representante público consegue realizar transações ilícitas com facilidade, considerando o cargo ou posição ocupada, visando auferir benefícios privados (Abramo, 2005). Nesse tipo de vínculo político, há um expressivo conluio entre gestores e políticos, resultando em práticas fraudulentas com o objetivo comum de obter benefícios particulares (Pan \& Tian, 2017).

O estreitamento das relações entre governo e empresas facilita as práticas ilegais de suborno. Embora a literatura ainda não tenha comprovado que as conexões políticas representam atos de corrupção, existem fortes indícios a partir dos benefícios próprios atrelados (Guo, 2018). A corrupção é considerada um importante canal para as corporações estabelecerem conexões políticas, devido aos privilégios envolvidos (Liu, Luo, \& Tian, 2016).

$\mathrm{H}_{2} \mathrm{~b}$ : Os conselheiros políticos diminuem a RSC das empresas na evidenciação de práticas anticorrupção.

\section{METODOLOGIA}

A amostra investigada foi composta por 372 empresas listadas na $[B]^{3}$ em 2016 e 368 em 2017; destas, 83 e 99 divulgaram relatório de sustentabilidade, respectivamente. Empresas constantes na amostra e que não divulgaram relatórios de sustentabilidade receberam nível de evidenciação igual a zero.

A escolha pelos relatórios de sustentabilidade para mensurar o nível de evidenciação das práticas anticorrupção deve-se ao fato de que as diretrizes da Global Reporting Initiative (GRI) possuem item específico para relatos de práticas anticorrupção.

A extensão dos relatórios sobre práticas anticorrupção é um indicador dos esforços da empresa em lidar com suborno e corrupção (Transparency International, 2009). A divulgação de práticas anticorrupção ajuda o aumento da consciência pública e coage as empresas para adotar princípios antiéticos (Hess, 2009).

Para mensurar a evidenciação de informações sobre práticas anticorrupção, procedeu-se pela busca e quantificação de expressões ou palavras-chave, apresentadas no Quadro 1.

\section{Quadro 1. Palavras-chave e expressões definidas}

Descrição

Anticorrupção; código de conduta; código de ética; combate à corrupção; compliance; corrupção; extorsão; Lava Jato; Lavagem de Dinheiro; Lei n. 12.846; propina; suborno(s); transparência.

Para confirmar que as palavras-chave refletem as práticas anticorrupção adotadas pelas empresas, foram analisadas práticas de coibição da corrupção encontradas nos relatórios de sustentabilidade diante das palavras-chave previamente definidas (Quadro 1). No Quadro 2, apresenta-se um exemplo de tal verificação. 


\section{Quadro 2. Práticas de coibição da corrupção encontradas nos relatórios de sustentabilidade diante da pesquisa pelas palavras-chave}

\begin{tabular}{l|l}
\hline Palavra-chave & Descrição encontrada \\
\hline \multirow{2}{*}{ Anticorrupção } & $\begin{array}{l}\text { O tema anticorrupção está presente nos processos de contratação de fornecedores, desde o momento da concorrência } \\
\text { quando divulgamos o Código de Conduta de Fornecedores, que possui questões baseadas nos princípios do Pacto } \\
\text { Global das Nações Unidas, até a formalização do contrato com cláusulas de Responsabilidade Socioambiental (Banco } \\
\text { Santander S.A., 2017). }\end{array}$ \\
\cline { 2 - 2 } &
\end{tabular}

Em 2017, o Conselho de Administração aprovou a revisão do Código de Conduta Ética. Adicionalmente, foram revisadas políticas e promovidos e-learnings obrigatórios a todos os colaboradores, com o intuito de disseminar a cultura anticorrupção e prevenção à lavagem de dinheiro (Cielo S.A., 2017).

A partir das palavras e expressões expostas no Quadro 1, foram auferidas 2.560 observações em 2016 e 3.621 em 2017 , nos relatórios investigados. Com o auxílio do software WordStat 8, foi possível buscar e quantificar as palavras-chave e expressões e, com o software FineCount, designar a quantidade de páginas dos relatórios de sustentabilidade, conforme métrica constante no Quadro 3.

\section{Quadro 3. Mensuração da evidenciação das práticas anticorrupção}

\begin{tabular}{l|l|l|l}
\hline Descrição & Métrica & Fonte & Autores de base \\
\hline $\begin{array}{l}\text { Evidenciação das Práticas } \\
\text { Anticorrupção (NEPA) }\end{array}$ & $\begin{array}{l}\text { Razão entre palavras-chaves pela quantidade } \\
\text { de páginas do relatório }\end{array}$ & $\begin{array}{l}\text { Relatórios de } \\
\text { sustentabilidade }\end{array}$ & $\begin{array}{l}\text { Gamerschlag, Möller e } \\
\text { Verbeeten (2011) }\end{array}$ \\
\hline
\end{tabular}

Para averiguar o reflexo dos mecanismos de GC na evidenciação voluntária das companhias, foram selecionadas as variáveis apontadas no Quadro 4.

\section{Quadro 4. Variáveis independentes de governança corporativa}

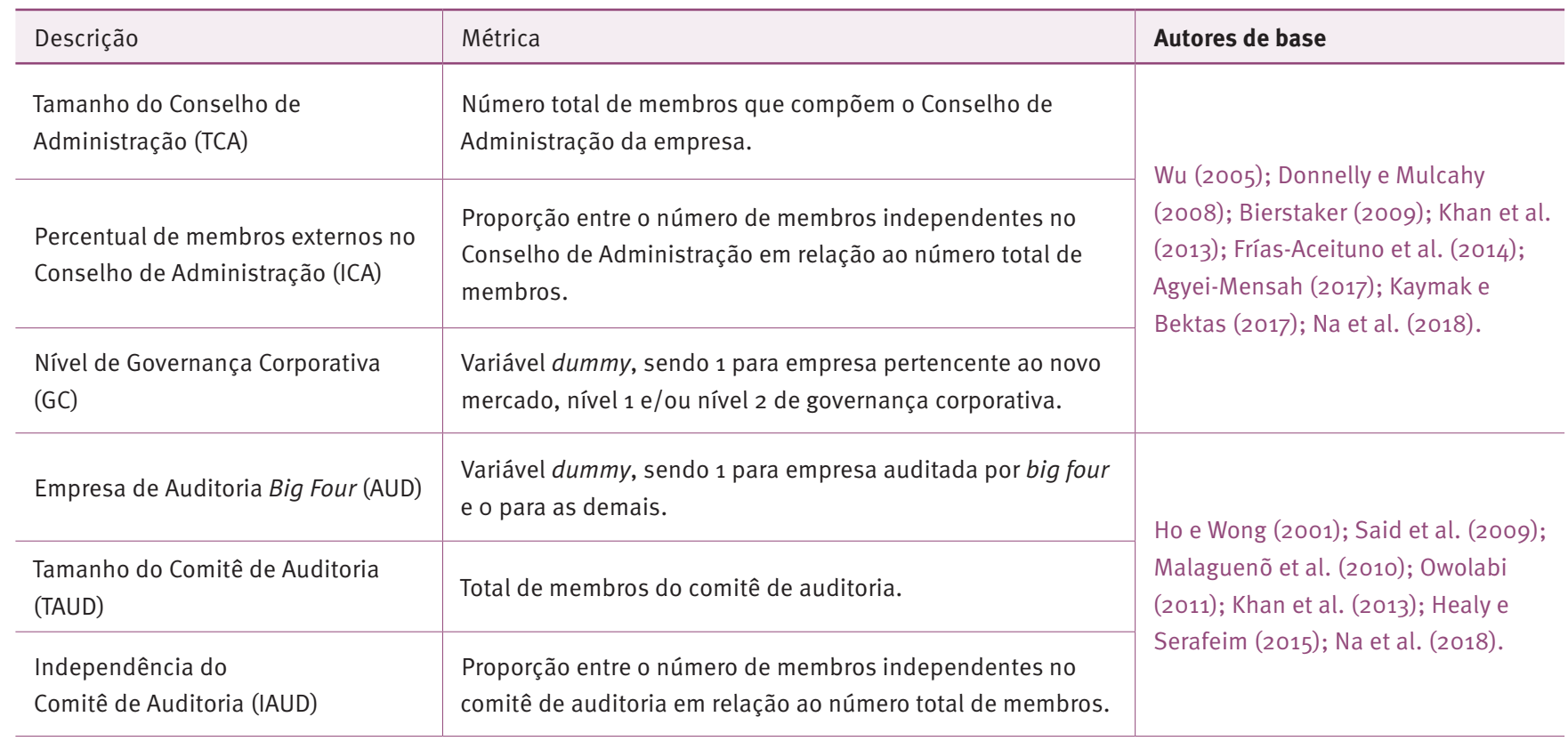

Para as conexões políticas, existentes entre empresas e governo, utilizaram-se as variáveis independentes demonstradas no Quadro 5. 


\section{Quadro 5. Variáveis independentes das conexões políticas}

\begin{tabular}{l|l|l}
\hline \multicolumn{1}{c|}{ Descrição } & \multicolumn{1}{c|}{ Métrica } & \multicolumn{1}{c}{ Autores de base } \\
\hline $\begin{array}{l}\text { Conexões políticas por meio de membros do } \\
\text { Conselho com expertise política (CPOL) }\end{array}$ & $\begin{array}{l}\text { Proporção de membros do Conselho de } \\
\text { Administração com expertise política. }\end{array}$ & $\begin{array}{l}\text { Camilo et al. (2012); Huang e Zhao (2016); } \\
\text { Wang et al. (2017). }\end{array}$ \\
\hline $\begin{array}{l}\text { Conexões políticas por meio de participação } \\
\text { acionária do governo (CPART) }\end{array}$ & $\begin{array}{l}\text { Variável dummy, sendo 1 se a empresa } \\
\text { possui participação acionária do governo } \\
\text { de maneira direta ou indireta e o para os } \\
\text { demais casos. }\end{array}$ & $\begin{array}{l}\text { Nasir e Abdullah (2004); Khan et al. (2013); } \\
\text { Huang e Zhao (2016); Said et al. (2009). }\end{array}$ \\
\hline
\end{tabular}

As características organizacionais também influenciam o nível informacional das empresas, sendo utilizadas como variáveis de controle (Quadro 6).

\section{Quadro 6. Variáveis de controle das características das empresas}

\begin{tabular}{|c|c|c|}
\hline Descrição & Métrica & Autores de base \\
\hline Tamanho (TAM) & $\begin{array}{l}\text { Logaritmo natural do valor contábil médio do } \\
\text { ativo total. }\end{array}$ & $\begin{array}{l}\text { Frías-Aceituno et al. (2014); Agyei-Mensah } \\
\text { (2017). }\end{array}$ \\
\hline Concentração de Propriedade (CP) & $\begin{array}{l}\text { Percentual de ações ordinárias em posse do } \\
\text { maior acionista. }\end{array}$ & Agyei-Mensah (2017); Na et al. (2018). \\
\hline Setor (SETOR) & $\begin{array}{l}\text { Variável dummy, sendo } 1 \text { para empresas } \\
\text { constantes nos setores de construção, } \\
\text { petróleo, gás e biocombustíveis e o para os } \\
\text { demais casos. }\end{array}$ & Frías-Aceituno et al. (2014); Na et al. (2018). \\
\hline Participação no ISE (ISE) & $\begin{array}{l}\text { Variável dummy, sendo } 1 \text { para empresas } \\
\text { pertencentes à carteira ISE e o para as demais. }\end{array}$ & Ricardo, Barcellos e Bortolon (2017). \\
\hline
\end{tabular}

Para analisar a influência de determinados mecanismos da GC e das conexões políticas na evidenciação de práticas anticorrupção, foram utilizadas a regressão linear múltipla e, complementarmente, a modelagem com efeitos fixos de ano e erropadrão robusto (Quadro 7). Também foram elaborados testes de sensibilidade contemplando apenas as empresas que divulgaram o relatório de sustentabilidade no período estudado.

\section{Quadro 7. Modelos econométricos}

$$
\begin{aligned}
& N E P A_{i t}=\beta_{0}+\beta_{1} T_{C A}+\beta_{i t} I C A_{i t}+\beta_{3} T_{A M}+\beta_{4} C P_{i t}+\beta_{5} S T_{i t}+\beta_{6} I S E_{i t}+\varepsilon \\
& N E P A_{i t}=\beta_{0}+\beta_{1} A U D_{i t}+\beta_{2} T A U D_{i t}+\beta_{3} I A U D_{i t}+\beta_{4} T A M_{i t}+\beta_{5} C P_{i t}+\beta_{6} S T_{i t}+\beta_{7} S_{i t}+\varepsilon \\
& N E P A_{i t}=\beta_{0}+\beta_{1} G_{i t}+\beta_{2} C P O L_{i t}+\beta_{2} C P A R T_{i t}+\beta_{3} T M_{i t}+\beta_{4} C P_{i t}+\beta_{5} S T_{i t}+\beta_{6} \mathrm{ISE}_{i t}+\varepsilon
\end{aligned}
$$

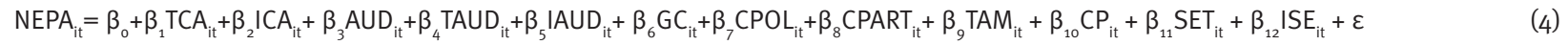

\section{RESULTADOS}

Entre as palavras-chave utilizadas, destacam-se “Combate à corrupção" e “Compliance”, que apresentaram aumentos expressivos de 2016 para 2017 (Tabela 1), resultado que se assemelha ao nível de evidenciação de práticas anticorrupção. 
Tabela 1. Observações por palavras-chave

\begin{tabular}{|c|c|c|c|c|c|c|c|c|c|c|c|c|c|c|}
\hline \multirow[b]{2}{*}{ Ano } & \multicolumn{2}{|c|}{ Anticorrupção } & \multicolumn{2}{|c|}{$\begin{array}{l}\text { Código de } \\
\text { conduta }\end{array}$} & \multicolumn{2}{|c|}{ Código de ética } & \multicolumn{2}{|c|}{$\begin{array}{l}\text { Combate à } \\
\text { corrupção }\end{array}$} & \multicolumn{2}{|c|}{ Compliance } & \multicolumn{2}{|c|}{ Corrupção } & \multicolumn{2}{|c|}{ Extorsão } \\
\hline & 2016 & 2017 & 2016 & 2017 & 2016 & 2017 & 2016 & 2017 & 2016 & 2017 & 2016 & 2017 & 2016 & 2017 \\
\hline Obs. & \multicolumn{2}{|c|}{$\begin{array}{c}\text { Lavagem de } \\
\text { dinheiro }\end{array}$} & \multicolumn{2}{|c|}{ Lava-Jato } & \multicolumn{2}{|c|}{$\begin{array}{c}\text { Lei } n \text {. } \\
12.846 / 2013\end{array}$} & \multicolumn{2}{|c|}{ Propina } & \multicolumn{2}{|c|}{ Suborno } & \multicolumn{2}{|c|}{ Transparência } & \multicolumn{2}{|c|}{ Total } \\
\hline Ano & 2016 & 2017 & 2016 & 2017 & 2016 & 2017 & 2016 & 2017 & 2016 & 2017 & 2016 & 2017 & 2016 & 2017 \\
\hline Obs. & 52 & 90 & 12 & 16 & 09 & 36 & 12 & 19 & 29 & 29 & 611 & 725 & 2560 & 3621 \\
\hline
\end{tabular}

A estatística descritiva das variáveis explicativas e de controle estão demonstradas na Tabela 2.

Tabela 2. Estatística descritiva e análise de frequência

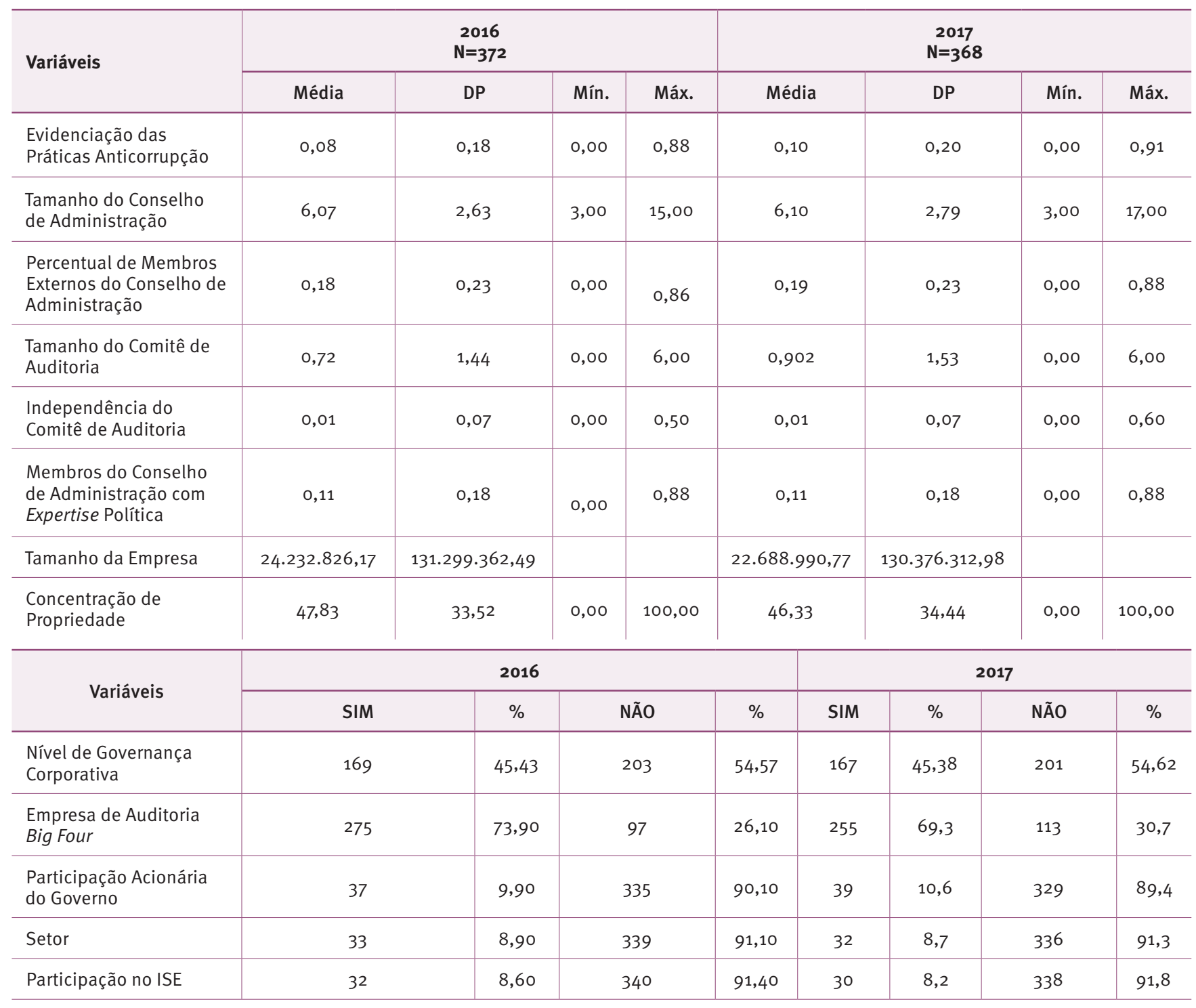


ARTIGOS | GOVERNANÇA CORPORATIVA E CONEXÕES POLÍTICAS NAS PRÁTICAS ANTICORRUPÇÃO

Sirlene Koprowski | Viviane Krein | Sady Mazzioni | Cristian Baú Dal Magro

Para analisar a influência das variáveis explicativas sobre o nível de evidenciação das práticas anticorrupção, foram utilizados modelos de regressão linear múltipla que consideraram variáveis de GC, auditoria e conexões políticas, separadamente (Tabela 3).

Tabela 3. Influência da governança corporativa e das conexões políticas na evidenciação de práticas anticorrupção

\begin{tabular}{|c|c|c|c|c|c|c|}
\hline \multirow{2}{*}{ Variáveis independentes } & \multicolumn{6}{|c|}{ Regressão linear com efeitos fixos de ano e erro-padrão robusto } \\
\hline & Modelo a & Modelo b & Modelo a & Modelo b & Modelo a & Modelo b \\
\hline Tamanho do Conselho de Administração & $0,007^{\star \star}$ & 0,0003 & & & & \\
\hline $\begin{array}{l}\text { Percentual de Membros Externos do Conselho de } \\
\text { Administração }\end{array}$ & $0,068^{\star *}$ & 0,0848 & & & & \\
\hline Empresa de Auditoria Big Four & & & $0,022 * *$ & 0,047 & & \\
\hline Tamanho do Comitê de Auditoria & & & $0,012^{\star \star}$ & $0,022^{\star \star}$ & & \\
\hline Independência do Comitê de Auditoria & & & 0,060 & $-0,101$ & & \\
\hline Nível de Governança Corporativa & & & & & $0,036 * \star$ & $-0,014$ \\
\hline Membros do Conselho com Expertise Política & & & & & $-0,012$ & $-0,173^{\star *}$ \\
\hline Participação Acionária do Governo & & & & & $0,101^{\star \star}$ & $0,125^{\star \star}$ \\
\hline Tamanho da Empresa & $0,041^{* *}$ & $0,049^{* *}$ & $0,042 * *$ & 0,029 & $0,041^{* *}$ & $0,046 * *$ \\
\hline Concentração de Propriedade & $0,001 * *$ & $0,001^{*}$ & $0,001 * *$ & 0,001 & $0,001 * *$ & 0,001 \\
\hline Setor & $-0,045^{\star *}$ & 0,108 & $-0,028$ & 0,165 & $-0,042^{\star *}$ & 0,153 \\
\hline Participação no ISE & $0,205^{\star *}$ & $0,063^{*}$ & $0,216^{\star \star}$ & $0,073^{\star \star}$ & $0,201^{\star \star}$ & $0,072^{\star \star}$ \\
\hline (Constante) & $-0,256 * *$ & $-0,052$ & $-0,237^{\star *}$ & 0,039 & $-0,225^{* *}$ & 0,010 \\
\hline Observações & 740 & 181 & 740 & 181 & 740 & 181 \\
\hline$R^{2}$ ajustado & 0,2918 & 0,1036 & 0,2897 & 0,1373 & 0,2989 & 0,1455 \\
\hline Prob > F. & $0,000^{\star \star}$ & $0,004^{\star \star}$ & $0,000^{* \star}$ & $0,001^{\star *}$ & $0,000^{\star *}$ & $0,000^{\star *}$ \\
\hline Breusch-pagan & 0,000 & 0,8952 & 0,000 & 0,6727 & 0,000 & 0,6292 \\
\hline Hausman & $0,032^{\star *}$ & $0,045^{\star \star}$ & $0,021^{\star \star}$ & $0,033^{* *}$ & $0,004^{\star *}$ & $0,008^{* *}$ \\
\hline Durbin Watson & 1,875 & 1,903 & 1,859 & 1,802 & 1,941 & 1,994 \\
\hline VIF & $<1,638$ & $<1,418$ & $<1,443$ & $\ll 1,353$ & $<1,620$ & $<1,599$ \\
\hline
\end{tabular}

a: teste com o total de observações; b: teste de sensibilidade excluindo as empresas que não publicaram o relatório de sustentabilidade.

**Significativo a $5 \%$; *Significativo a $10 \%$.

Salienta-se que as regressões classificadas no modelo “a” contemplam todas as empresas da amostra e, no modelo "b", apenas as empresas que divulgaram os relatórios de sustentabilidade. Todos os modelos foram elaborados a partir da regressão linear múltipla e, posteriormente, foi feito o controle de efeitos fixos de ano e erro-padrão robusto, sendo apenas os últimos divulgados na Tabela 3, pois a variação dos coeficientes e da significância não apontou diferenças relevantes nos resultados. Comprova-se a adequação das regressões ao modelo de efeitos fixos em decorrência de o teste de Hausman ter apresentado significância estatística ao nível de 5\%.

Os achados evidenciam que as empresas com CA maiores e com maior percentual de membros externos são mais propensas à evidenciação das práticas anticorrupção. Os resultados são consistentes com Agyei-Mensah (2017), ao constatar que maior proporção de conselheiros independentes estimula a divulgação voluntária. Comprova-se que membros externos no CA apresentam maior comprometimento e sensibilidade nas questões éticas 
e maior interesse em atender aos anseios das diversas partes interessadas, contribuindo também com o combate à corrupção (Frías-Aceituno et al., 2014).

Complementarmente, o menor vínculo entre membros externos e demais integrantes do CA dificulta práticas de conluio e aumenta a sensação de fiscalização. Considerando os casos de corrupção noticiados pela mídia nacional, torna-se plausível presumir que os membros externos do CA têm dado especial atenção ao combate à corrupção (Luca, Moura, \& Nascimento, 2012).

Das características de auditoria, o fato de a empresa ser auditada por firma big four e o tamanho do comitê de auditoria se mostraram significativos, permitindo inferir que companhias com tais condições apresentam maior volume de evidenciação de práticas anticorrupção. 0 resultado é consistente com os achados de Malagueño et al. (2010), Owolabi (2011), Healy e Serafeim (2015) e Na et al. (2018), revelando que a qualidade da auditoria aumenta as práticas anticorrupção.

As evidências permitem argumentar que os mecanismos de GC utilizados no estudo se refletem na RSC das empresas pela evidenciação de práticas anticorrupção. Comprova-se que a RSC no combate à corrupção tem sido exercida pela atuação dos mecanismos de GC, corroborando as evidências anteriores de Wu (2005), Agyei-Mensah (2017), Hills et al. (2009), Owolabi (2011), Branco \& Delgado (2012), Hoi \& Lin (2012), Frías-Aceituno et al. (2014) e Na et al. (2018). Além disso, a GC tem utilizado a RSC para minimizar os conflitos de agência, pela divulgação de práticas anticorrupção, conforme sugerido por Malagueño et al. (2010). Isso aumenta a confiança entre controlador e minoritários perante as decisões corporativas (Jensen \& Meckling, 1976).

Sugere-se que a conexão política, pela participação do governo como acionista, influencia positivamente a evidenciação das práticas anticorrupção, comprovando-se as evidências de melhoria da RSC (Etxeberria \& Odriozola, 2018; Huang \& Zhao, 2016; Khan et al., 2013; Nasir \& Abdullah, 2004; Said et al., 2009).

As empresas com participação acionária do governo zelaram pela reputação corporativa e pelo atendimento aos stakeholders, evidenciando informações mais qualificadas sobre práticas anticorrupção (Etxeberria \& Odriozola, 2018), confirmando os preceitos da Teoria da Agência. A presença do governo na estrutura de propriedade das empresas influenciou o maior repasse informacional sobre medidas de combate à corrupção, devido a certa obrigação moral que possui perante a sociedade, principalmente, a partir da promulgação da Lei $n$. $12.846 / 2013$.

Por sua vez, a participação de políticos no CA influencia negativamente a evidenciação das práticas anticorrupção. Os achados sugerem que os interesses compensatórios das empresas que estreitam vínculos com políticos no CA podem ser um fator que limita a evidenciação de práticas anticorrupção. Além disso, o benefício privado do político que ocupa assento no CA faz com que o monitoramento corporativo seja prejudicado (Camilo et al., 2012; Chaney et al., 2011; Guedhami et al., 2014).

Quanto às variáveis de controle, o tamanho da empresa apresentou influência positiva nas práticas anticorrupção. 0 resultado é condizente com o pressuposto de que as empresas maiores estão expostas à avaliação e julgamento público, evidenciando informações voluntárias em maior quantidade (Agyei-Mensah, 2017; Frías-Aceituno et al., 2014). O recente contexto político e empresarial brasileiro justifica a postura das grandes empresas em assuntos de combate à corrupção, visando legitimar sua atuação no mercado.

A participação da empresa na carteira ISE contribuiu no nível de evidenciação das práticas de combate à corrupção. O fato de uma determinada empresa compor tal carteira é derivado de iniciativas proativas em relação às questões sociais, incluindo o combate à corrupção. Essa agenda, possivelmente, foi impulsionada após a promulgação da Lei n. 12.846/2013, e as empresas que compõem o ISE ressaltaram suas práticas contra a corrupção, em busca de melhor reputação organizacional. Assim, a adoção da RSC alia os interesses sociais às estratégias das organizações (Hills et al., 2009).

As empresas dos setores de construção, petróleo, gás e biocombustíveis apresentaram uma relação negativa com a evidenciação de práticas anticorrupção. As empresas desses setores de atuação foram amplamente citadas nos escândalos de corrupção da Operação Lava Jato e evidenciaram com menor afinco as informações referentes às iniciativas das companhias no combate à corrupção. 0 resultado encontra consistência no pressuposto de que quanto maior a exposição da organização em casos de corrupção, menor será seu repasse informacional referente às medidas de prevenção (Barkemeyer, Preuss, \& Lee, 2015).

A maior concentração de propriedade das empresas, embora tenha apresentado significância estatística em alguns modelos, demonstrou coeficientes muito baixos de influência no nível de evidenciação de informações para o combate à corrupção, cuja força explicativa deve ser considerada com parcimônia. Contudo, os resultados vão ao encontro de Braga, Oliveira e Salotti (2009) e Murcia e Santos (2009), refutando o pressuposto de que a propriedade acionária diluída aumenta a divulgação voluntária para reduzir o conflito de agência, preconizado pela Teoria da Agência.

Por fim, foram elaborados testes de sensibilidade utilizando-se equações com os mecanismos de GC, conexões 
ARTIGOS | GOVERNANÇA CORPORATIVA E CONEXÕES POLÍTICAS NAS PRÁTICAS ANTICORRUPÇÃO

Sirlene Koprowski | Viviane Krein | Sady Mazzioni | Cristian Baú Dal Magro

políticas e as variáveis de controle, conjuntamente. Alguns testes consideraram todas as empresas da amostra e outros, apenas aquelas que divulgaram relatórios de sustentabilidade. Os modelos utilizaram regressão linear múltipla, controle de efeitos fixos de ano e erro-padrão robusto.

Tabela 4. Teste de sensibilidade da influência da governança corporativa e das conexões políticas na evidenciação de práticas anticorrupção

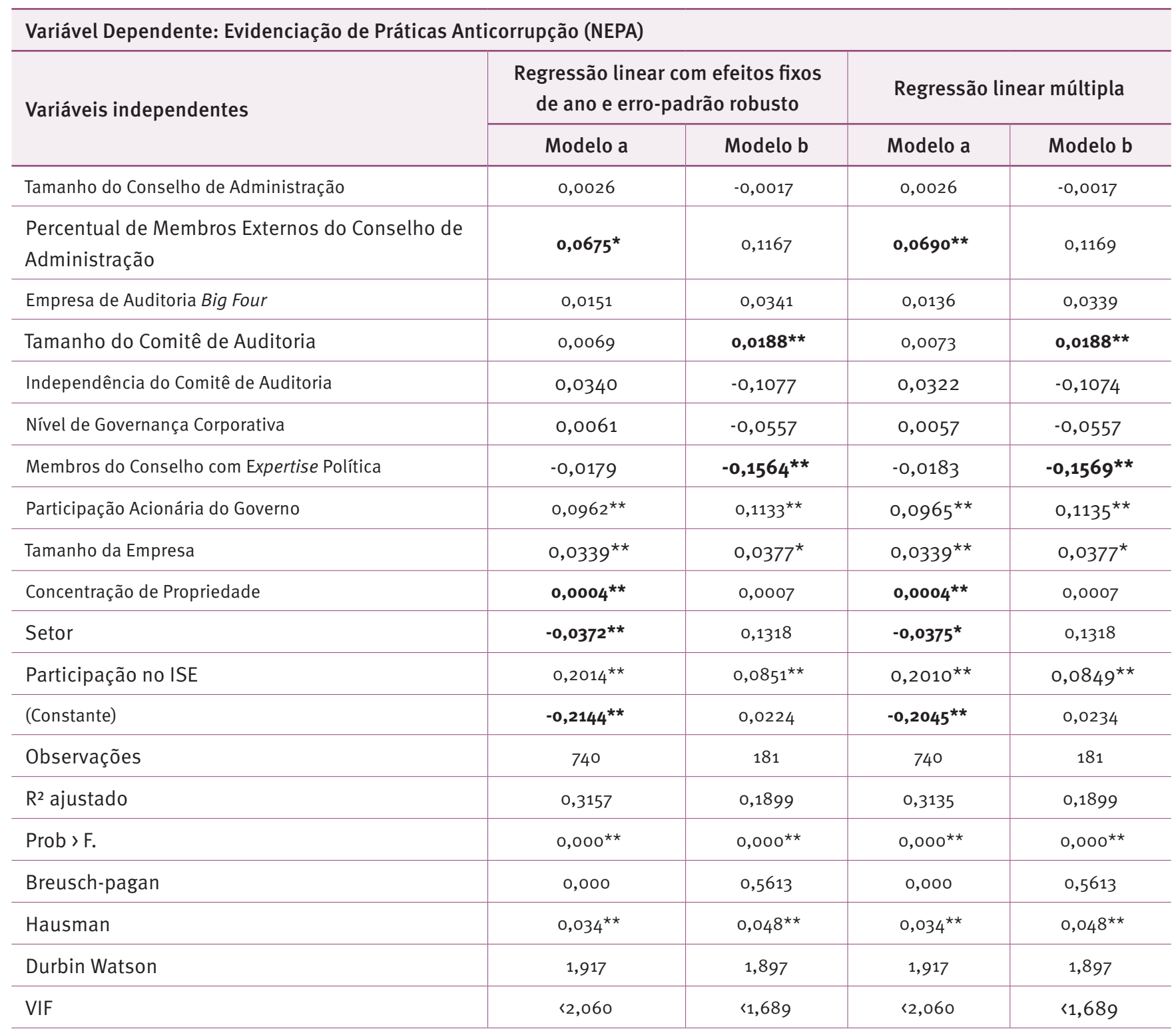

a: teste com o total de observações; b: teste de sensibilidade excluindo as empresas que não publicaram o relatório de sustentabilidade.

**Significativo a $5 \%$; *Significativo a $10 \%$.

Os resultados suportam a tendência de que a presença de políticos no CA faz com que as empresas tenham uma menor preocupação com a divulgação de práticas anticorrupção, corroborando outras evidências de Abramo (2005), Camilo et al. (2012), Chaney et al. (2011), Guedhami et al. (2014), Liu et al. (2016) e Guo (2018). Confirmou-se, pelas evidências, que a participação acionária do governo provocou aumento na evidenciação de práticas anticorrupção pelas empresas. Além disso, reforçam que maior percentual de membros independentes no CA e o tamanho do comitê de auditoria impactam o aumento das práticas anticorrupção. 


\section{CONSIDERAÇÕES FINAIS}

No âmbito da estrutura de propriedade, a presença do governo como acionista, em complemento aos mecanismos de GC, ampliou o envolvimento das empresas no combate à corrupção, encorajando a promoção da RSC pelas práticas anticorrupção. Esse resultado é decorrente da constante pressão das partes interessadas no ambiente nacional, tendo em vista os escândalos corporativos com o envolvimento de políticos. Os efeitos práticos são a mitigação dos conflitos de agência, a melhoria da RSC e a redução da assimetria informacional perante as partes interessadas.

Por sua vez, a presença de políticos no CA faz com que o engajamento com a RSC seja prejudicado, reduzindo as preocupações com as práticas anticorrupção. Esse resultado oferece insights às evidências de conflitos de interesses ocasionados pela falha no monitoramento da GC em função da presença de políticos nas empresas (Ding et al., 2015; Huang \& Zhao, 2016; Pan \& Tian, 2017).

Os conselheiros políticos apresentam menor preocupação com a adoção de práticas de combate à corrupção, fazendo com que as partes interessadas da firma tenham que observar o comportamento dessas empresas com maior parcimônia. 0 efeito prático é a assimetria informacional, que ocasiona conflitos de agência, prejudica o engajamento na RSC e a atuação da empresa no combate à corrupção.

Sugere-se que, no campo da GC, a presença de políticos no CA agrava os conflitos de interesses entre majoritários e minoritários (Ding et al., 2015; Huang \& Zhao, 2016; Pan \& Tian, 2017), contrariando a aliança entre conexão política e GC no fortalecimento da imagem corporativa, conforme sugerido por Said et al. (2009). A legitimação só acontece quando o governo faz parte da estrutura de propriedade das organizações, em que a imagem corporativa parece ser fundamental às partes interessadas.

Conclui-se que a participação acionária do governo apresenta maior interesse na manutenção da reputação das empresas, tendo em vista que as partes interessadas vinculam com facilidade o governo e a empresa. Por sua vez, políticos no CA apresentam interesses divergentes, pelo fato de sua presença estar oculta à maioria das partes interessadas e facilitar práticas de corrupção pela obtenção de benefícios privados.

O estudo buscou diferenciar-se pela forma de análise da corrupção, tendo em vista que poucas pesquisas empíricas avaliam esse problema social sob o ponto de vista da divulgação pelas empresas. Contudo, deve-se observar que o fato de as empresas evidenciarem termos relacionados com as práticas anticorrupção não as exime de possíveis desconformidades, mas o tema tem ocupado a agenda de preocupações dos executivos.
Entre as limitações, pode-se considerar a ausência da característica de dualidade de CEO, ou seja, a mesma pessoa ocupar os cargos de presidência da empresa e presidência do CA. Essa limitação constitui-se em perspectiva para pesquisas futuras adicionarem esse elemento na investigação. A continuidade das investigações pode considerar, ainda, elementos como a natureza do acionista controlador e a distribuição de dividendos.

\section{NOTA DE AGRADECIMENTO}

\section{Agradecemos à PROSUC/CAPES.}

\section{REFERÊNCIAS}

Abramo, C. W. (2005). Percepções pantanosas: A dificuldade de medir a corrupção. Novos Estudos CEBRAP, (73), 33-37.

Aguilera, R. V., Rupp, D. E., Williams, C. A., \& Ganapathi, J. (2007) Putting the $S$ back in corporate social responsibility: A multilevel theory of social change in organizations. Academy of Management Review, 32(3), 836-863. doi:10.2307/20159338

Agyei-Mensah, B. K. (2017). The relationship between corporate governance, corruption and forward-looking information disclosure: A comparative study. Corporate Governance: The International Journal of Business in Society, 17(2), 284-304. doi: 10.1108/CG-112015-0150

Barkemeyer, R., Preuss, L., \& Lee, L. (2015). Corporate reporting on corruption: An international comparison. Accounting Forum, 39(4), 349-365. doi: 10.1016/j.accfor.2015.10.001

Bierstaker, J.L.(2009). Differences in attitudes aboutfraud and corruption across cultures: Theory, examples and recommendations. Cross Cultural Management: An International Journal, 16(3), 241-250. doi: 10.1108/13527600910977337

Bishara, N. D. (2011). Governance and corruption constraints in the Middle East: Overcoming the business ethics glass ceiling. American Business Law Journal, 48(2), 227-283. doi:10.1111/j.17441714.2011.01115.x

Boubakri, N., Cosset, J. C., \& Saffar, W. (2008). Political connections of newly privatized firms. Journal of Corporate Finance, 14(5), 654-673. doi: 10.1016/j.jcorpfin.2008.08.003

Braga, J. P., Oliveira, J. R. S., \& Salotti, B. M. (2009). Determinantes do nivel de divulgação ambiental nas demonstrações contábeis de empresas brasileiras. Revista de Contabilidade UFBA, 3(3), 81-95.

Branco, M. C., \& Delgado, C. (2012). Business, social responsibility, and corruption. Journal of Public Affairs, 12 (4), 357-365. doi: 10.1016/j. jcae.2018.02.002

Camilo, S. P. O., Marcon, R., \& Bandeira-de-Mello, R. (2012). Conexões políticas das firmas e seus efeitos na performance: Uma convergência entre as perspectivas da governança e da dependência de recursos um ensaio teórico. Revista Alcance, 19(2), 241-258. doi: 10.14210/ alcance.v19n2.p241-258 
Castro, P. R., Amaral, J. V., \& Guerreiro, R. (2019). Adherence to the compliance program of Brazil's anti-corruption law and internal controls implementation. Revista Contabilidade \& Finanças, 30(80), 186-201. doi: 10.1590/1808-057x201806780

Chaney, P. K., Faccio, M., \& Parsley, D. (2011). The quality of accounting information in politically connected firms. Journal of Accounting and Economics, 51(1-2), 58-76. doi: 10.1016/j.jacceco.2010.07.003

Chow, C., \& Wong-Boren, A. (1987). Voluntarily disclosure by Mexican companies. The Accounting Review, 62, 533-541.

Claessens, S., Feijen, E., \& Laeven, L. (2008). Political connections and preferential access to finance: The role of campaign contributions. Journal of Financial Economics, 88(3), 554-580. doi: 10.1016/j.jfineco.2006.11.003

Correia, M. M. (2014). Political connections and SEC enforcement. Journal of Accounting and Economics, 57(2-3), 241-262. doi: 10.1016/j. jacceco.2014.04.004

Dami, A. B. T., Rogers, P., \& Ribeiro, K. C. S. (2007). Estrutura de propriedade no Brasil: Evidências empíricas do grau de concentração acionária. Contextus: Revista Contemporânea de Economia e Gestão, 5(2), 21-30.

Depoers, F. (2000). A cost benefit study of voluntarily disclosure: Some empirical evidence from French listed companies. European Accounting Review, 9, 245-263.

Ding, S., Jia, C., Wilson, C., \& Wu, Z. (2015). Political connections and agency conflicts: The roles of owner and manager political influence on executive compensation. Review of Quantitative Finance and Accounting, 45(2), 407-434.

Donnelly, R., \& Mulcahy, M. (2008). Board structure, ownership, and voluntary disclosure in Ireland. Corporate Governance: An International Review, 16(5), 416-429. doi: 10.1111/j.14678683.2008.00692.x

Etxeberria, I. A., \& Odriozola, M. A. (2018). The social reputation of European companies: Does anti-corruption disclosure affect stakeholders' perceptions? Corporate Social Responsibility and Environmental Management, 25(5), 713-721. doi: 10.1002/csr.1488

Frías-Aceituno, J. V., Rodríguez-Domínguez, L., \& García-Sánchez, I. M. (2014). Políticas contra la corrupción y el soborno: Se involucran de manera similar los consejeros en diferentes entornos corporativos? Revista Europea de Dirección y Economía de la Empresa, 23(1), 31-42. doi: 10.1016/j.redee.2013.09.004

Gamerschlag, R., Möller, K., \& Verbeeten, F. (2011). Determinants of voluntary CSR disclosure: Empirical evidence from Germany. Review of Managerial Science, 5(2-3), 233-262. doi:10.1007/s11846-010$0052-3$

Guedhami, O., Pittman, J. A., \& Saffar, W. (2014). Auditor choice in politically connected firms. Journal of Accounting Research, 52(1), 107-162. doi: 10.1111/1475-679X.12032

Guo, C. (2018). The impact of the anti-corruption campaign on the relationship between political connections and preferential bank loans: The case of China. Emerging Markets Finance and Trade, 54(11), 1-48. doi: 10.1080/1540496X.2018.1454306

Halter, M. V., Arruda, M. C. C., \& Halter, R. B. (2009). Transparency to reduce corruption? Journal of Business Ethics, 84(3), 373-385. doi: 0.1007/s10551-009-0198-6
Healy, P. M., \& Serafeim, G. (2015). An analysis of firms self-reported anticorruption efforts. The Accounting Review, 91(2), 489-511. doi: $10.2308 /$ accr-51191

Hess, D. (2009). Catalyzing corporate commitment to combating corruption. Journal of Business Ethics, 88(4), 781-790. doi: 10.1007/ s10551-009-0322-7

Hills, G., Fiske, L., \& Mahmud, A. (2009). Anti-corruption as strategic CSR: A call to action for corporations. FSG Social Impact Advisors, May, 1-52. Retrieved from http://nncg.issuelab.org/resources/2435/2435.pdf

Ho, S. S., \& Wong, K. S. (2001). A study of the relationship between corporate governance structures and the extent of voluntary disclosure. Journal of International Accounting, Auditing and Taxation, 10(2), 139-156. doi: 10.1016/S1061-9518(01)00041-6

Hoi, Y. H., \& Lin, C. Y. (2012). Preventing corporate corruption: The role of corporate social responsibility strategy. International Journal Business Behavior Science, 2(1), 12-22. Retrieved from http://www. csringreece.gr/files/research/CSR-1331563230.pdf

Huang, H., \& Zhao, Z. (2016). The influence of political connection on corporate social responsibility-evidence from listed private companies in China. International Journal of Corporate Social Responsibility, 1(1), 1-9. doi: 10.1186/s40991-016-0007-3

Jamali, D., Safieddine, A. M., \& Rabbath, M. (2008). Corporate governance and corporate social responsibility synergies and interrelationships. Corporate Governance: An International Review, 16(5), 443-459. doi: 10.1111/j.1467-8683.2008.00702.x

Jensen, M. C., \& Meckling, W. H. (1976). Theory of the firm: Managerial behavior, agency costs and ownership structure. Journal of Financial Economics, 3(4), 305-360. doi: 10.1016/0304-405X(76)90026-X

Jo, H., \& Harjoto, M. A. (2012). The causal effect of corporate governance on corporate social responsibility. Journal of Business Ethics, 106(1), 53-72. doi:10.1007/s10551-011-1052-1

Joseph, C., Gunawan, J., Sawani, Y., Rahmat, M., Noyem, J. A., \& Darus, F. (2016). A comparative study of anti-corruption practice disclosure among Malaysian and Indonesian Corporate Social Responsibility (CSR) best practice companies. Journal of Cleaner Production, 112, 2896-2906. doi: 10.1016/j.jclepro.2015.10.091

Kaymak, T., \& Bektas, E. (2017). Corporate social responsibility and governance: Information disclosure in multinational corporations. Corporate Social Responsibility and Environmental Management, 24(6), 555-569. doi: 10.1002/csr.1428

Khan, A., Muttakin, M. B., \& Siddiqui, J. (2013). Corporate governance and corporate social responsibility disclosures: Evidence from an emerging economy. Journal of Business Ethics, 114(2), 207-223. doi: 10.1007/s10551-012-1336-0

Krishnamurti, C., Shams, S., \& Velayutham, E. (2018). Corporate social responsibility and corruption risk: A global perspective. Journal of Contemporary Accounting \& Economics, 14(1), 1-21. doi: 10.1016/j. jcae.2018.02.002

Lei n. 12.846, de $1^{10}$ de agosto de 2013. (2013). Dispõe sobre a responsabilização administrativa e civil de pessoas jurídicas pela prática de atos contra a administração pública, nacional ou estrangeira, e dá outras providências. Recuperado de http://www. planalto.gov.br/ccivil_03/_ato2011-2014/2013/lei/l12846.htm

Leuz, C. (2006). Cross listing, bonding and firms reporting incentives: A discussion of Lang, Raedy and Wilson. Journal of Accounting and Economics, 42, 285-299. 
Liu, Q., Luo, T., \& Tian, G. (2016). Political connections with corrupt government bureaucrats and corporate M\&A decisions: A natural experiment from the anti-corruption cases in China. Pacific-Basin Finance Journal, 37, 2-80. doi: 10.1016/j.pacfin.2016.03.003

Luca, M. M. M., Moura, A. A. F., \& Nascimento, F. S. P. (2012). Evidenciação voluntária de informações sociais por empresas de capital aberto no Brasil com base nos indicadores de responsabilidade social da ONU. Revista Alcance, 19(3), 362-380. doi: 10.14210/alcance.v19n3.p362380

Malagueño, R., Albrecht, C., Ainge, C., \& Stephens, N. (2010). Accounting and corruption: A cross-country analysis. Journal of Money Laundering Control, 13(4), 372-393. doi: 10.1108/13685201011083885

Martynova, M., \& Renneboog, L. (2009). What determines the financing decision in corporate takeovers: Cost of capital, agency problems, or the means of payment? Journal of Corporate Finance, 15(3), 290-315. doi: 10.1016/j.jcorpfin.2008.12.004

Murcia, F.D.-R., \& Santos, A. (2009). Fatores determinantes do nível de disclosure voluntário das companhias abertas no Brasil. Revista de Educação e Pesquisa em Contabilidade, 3(2), 72-95.

Na, K., Kang, Y. H., \& Kim, Y. (2018). The effect of corporate governance on the corruption of firms in BRICs (Brazil, Russia, India \& China). Social Sciences, 7(6), 1-16. doi: 10.3390/socsci7060085

Nasir, N. M., \& Abdullah, S. N. (2004). Voluntary disclosure and corporate governance among financially distressed firms in Malaysia. Financial Reporting, Regulation and Governance, 3(1), 1-39.

Nassif, E., \& Souza, C. L. (2013). Conflitos de agência e governança corporativa. CAD-Caderno de Administração, 7(1), 1-20.

Owolabi, A. (2011). Corruption and the environment of accounting and auditing in Africa. International Journal of Critical Accounting, 3(2-3), 220-234. doi: 10.1504/IJCA.2011.039752

Padula, A. J. A., \& Albuquerque, P.H. M. (2018). Corrupção governamental no mercado de capitais: Um estudo acerca da operação Lava Jato. RAE-Revista de Administração de Empresas, 58(4), 405-417. doi: 10.1590/s0034-759020180406

Pan, X., \& Tian, G. G. (2017). Political connections and corporate investments: Evidence from the recent anti-corruption campaign in China. Journal of Banking \& Finance, 11(40), 1-15. doi: 10.1016/j. jbankfin.2017.03.005
Polsiri, P., \& Jiraporn, P. (2012). Political connections, ownership structure, and financial institution failure. Journal of Multinational Financial Management, 22(1-2), 39-53. doi: 10.1016/j.mulfin.2012.01.001

Raffournier, B. (1995). The determinants of voluntary disclosure by Swiss Listed Companies. European Accounting Review, 4(2), 261-280. doi: $10.1080 / 09638189500000016$

Ricardo, V. S., Barcellos, S. S., \& Bortolon, P. M. (2017). Relatório de sustentabilidade ou relato integrado das empresas listadas na BM\&FBovespa: Fatores determinantes de divulgação. Revista de Gestão Social e Ambiental, 11(1), 90-104. doi: 10.24857/rgsa. v11i1.1233

Rodriguez, P., Siegel, D. S., Hillman, A., \& Eden, L. (2006). Three lenses on the multinational enterprise: Politics, corruption, and corporate social responsibility. Journal of International Business Studies, 376, 733-746. doi: 10.2139/ssrn.900544

Said, R., Zainuddin, Y. H., \& Haron, H. (2009). The relationship between corporate social responsibility disclosure and corporate governance characteristics in Malaysian public listed companies. Social Responsibility Journal, 5(2), 212-226. doi:https://doi. org/10.1108/17471110910964496

Silveira, A. D., \& Barros, L. A. B. C. (2008). Determinantes da qualidade da governança corporativa das companhias abertas brasileiras. Revista Eletrônica de Administração, 14(3), 512-540.

Transparência Internacional. (2017). Índice de percepção da corrupção 2017. Recuperado de https://www.ipc.transparenciainternacional. org.br/

Transparency International. (2009). Transparency in reporting on anticorruption: A report on corporate practices. Retrieved from https:// www.transparency.org/whatwedo/publication/transparency_in_ reporting_on_anti_corruption_a_report_on_corporate_practice

Wang, Z., Chen, M. H., Chin, C. L., \& Zheng, Q. (2017). Managerial ability, political connections, and fraudulent financial reporting in China. Journal of Accounting and Public Policy, 36(2), 141-162. doi: 10.1016/j.jaccpubpol.2017.02.004

$\mathrm{Wu}, \mathrm{X}$. (2005). Corporate governance and corruption: A cross country analysis. Governance, 18(2), 151-170. doi: 10.1111/j.1468$0491.2005 .00271 . x$

\section{CONTRIBUIÇÃO DOS AUTORES}

Os autores declaram que participaram de forma conjunta da conceitualização e abordagem teórica-metodológica, revisão teórica (levantamento de literatura), análise de dados e, por fim, da redação e revisão final. A coleta de dados foi realizada pelas autoras Sirlene Koprowski e Viviane Krein. 